\title{
TNF-related apoptosis-inducing ligand (TRAIL) protects against diabetes and atherosclerosis in $\mathrm{Apoe}^{-/-}$mice
}

\author{
B. A. Di Bartolo • J. Chan • M. R. Bennett • \\ S. Cartland $\cdot$ S. Bao $\cdot$ B. E. Tuch $\cdot$ M. M. Kavurma
}

Received: 19 July 2011 / Accepted: 9 August 2011 / Published online: 1 October 2011

(C) Springer-Verlag 2011

\begin{abstract}
Aims/hypothesis TNF-related apoptosis-inducing ligand (TRAIL) is implicated in the regulation of diabetes and is reduced in patients with cardiovascular disease. Although TRAIL receptors are widespread, and TRAIL can promote cell proliferation and apoptosis, it is not known how TRAIL might protect against diabetes and atherosclerosis.

Methods We examined the development of atherosclerosis and diabetes in Apoe ${ }^{-/}$, Trail (also known as Tnfsf10) ${ }^{-/}$Apoe $^{-/}$ and $\mathrm{Trail}^{/}$mice that were fed a high-fat diet. Plasma cholesterol, triacylglycerol, glucose and insulin, as well as changes in various metabolic enzymes and regulators were assessed. Glucose and insulin tolerance tests were performed. Pancreatic islets were examined for insulin and beta cell dysfunction (apoptosis and macrophage infiltration).

Results Compared with Apoe $e^{-/-}$mice, Trail ${ }^{\prime-}$ Apoe $^{-/-}$and $\mathrm{Trai}^{\prime-}$ mice exhibited several features of diabetes, including increased weight, hyperglycaemia, reduced plasma
\end{abstract}

Electronic supplementary material The online version of this article (doi:10.1007/s00125-011-2308-0) contains peer-reviewed but unedited supplementary material, which is available to authorised users.

B. A. Di Bartolo $\cdot$ J. Chan $\cdot$ S. Cartland $\cdot$ M. M. Kavurma $(\square)$ Centre for Vascular Research, University of New South Wales, Sydney NSW 2052, Australia

e-mail:m.kavurma@unsw.edu.au

M. R. Bennett

Division of Cardiovascular Medicine, University of Cambridge,

Cambridge, UK

B. E. Tuch

CSIRO Materials Science and Engineering,

Ryde, NSW, Australia

S. Bao

Discipline of Pathology, University of Sydney,

Sydney, NSW, Australia insulin, impaired glucose tolerance, beta cell dysfunction, reduced islet insulin, macrophage infiltration and increased apoptosis. Trail ${ }^{/-}$Apoe $^{-/-}$mice had increased plasma cholesterol, triacylglycerol, and VLDL- and LDLcholesterol, and increased expression of genes involved in cholesterol synthesis and lipogenesis. Trail ${ }^{-1}$ Apoe $^{-/-}$mice also had increased atherosclerosis, with several features of plaque instability.

Conclusions/interpretation We show for the first time that TRAIL deficiency promotes numerous features of diabetes that are typical of human disease, and are associated with reduced insulin and pancreatic inflammation/apoptosis. TRAIL also regulates cholesterol and triacylglycerol homeostasis in Apoe $^{-/}$mice by increasing the expression of genes involved in (1) cholesterol synthesis and absorption, and (2) triacylglycerol production.

Keywords Atherosclerosis · Diabetes · TRAIL

$\begin{array}{ll}\text { Abbreviations } \\ \text { ApoE } & \text { Apolipoprotein E } \\ \text { CVD } & \text { Cardiovascular disease } \\ \text { HOMA-B } & \text { HOMA of beta cell function } \\ \text { MCP-1 } & \text { Monocyte chemoattractant protein-1 } \\ \text { MHC } & \text { Major histocompatibility complex } \\ \text { PCNA } & \text { Proliferating cell nuclear antigen } \\ \text { SMA } & \text { Smooth muscle- } \alpha \text { actin } \\ \text { TRAIL } & \text { TNF-related apoptosis-inducing ligand } \\ \text { VSMC } & \text { Vascular smooth muscle cell }\end{array}$

Introduction

TNF-related apoptosis-inducing ligand (TRAIL), a member of the TNF superfamily, induces apoptosis and proliferation 
of some cell types [1-3]. In humans, TRAIL induces apoptosis following engagement with death receptor-4 and -5 . Decoy receptor-1 and -2 , and osteoprotegerin, which regulates osteoclastogenesis by binding the receptor activator of nuclear factor $\mathrm{kB}$ ligand, can compete with the death receptors and protect cells from apoptosis. TRAIL can also promote activation of extracellular signal-regulated kinase 1/2, mitogen-activated protein kinase, c-Jun Nterminal kinase and Akt, pathways that are involved in survival and proliferation of cells $[2,4]$.

TRAIL deficiency has been associated with autoimmune diabetes in a mouse model of human type 1 diabetes. For example, the antagonisation of TRAIL signalling by injection of the soluble TRAIL receptor into non-obese diabetic mice or streptozotocin-treated $\mathrm{Trail}^{\prime-}$ mice increased the incidence of diabetes compared with controls $[5,6]$. In cardiovascular disease (CVD), Trail $^{\prime-}$ mice were protected from intimal thickening following vascular arterial injury [1]; moreover, in diabetic Apoe $^{-/-}$mice TRAIL stabilised atherosclerotic plaque by increasing vascular smooth muscle cell (VSMC) content, and promoted apoptosis of inflammatory cells [7]. Indeed, circulating TRAIL levels tend to be lower in patients with diabetes, and significantly reduced in patients with CVD $[8,9]$. Thus, TRAIL may be an important protective factor in diabetes and atherosclerosis. However, mechanisms of its regulatory role remain unexplored.

Apolipoprotein E (ApoE)-deficient $\left(A p o e^{-/}\right)$mice are unable to produce the key glycoprotein ApoE essential for transport and metabolism of lipids. Compared with normal mice, Apoe $e^{-/-}$mice have markedly altered lipoprotein profiles and rapidly develop atherosclerosis [10]. In this study, we examined the role of TRAIL in diabetes and atherosclerosis by generating $\mathrm{Trail}^{-1} \mathrm{Apoe}^{-/-}$mice. On a 'Western' high-fat diet for 12 weeks, $\mathrm{Trail}^{-1} \mathrm{Apoe}^{-/-}$mice displayed features of diabetes, including increased body weight and adiposity, increased plasma glucose, reduced plasma insulin, increased islet inflammation and apoptosis, impaired glucose tolerance, and ketogenesis. These mice had increased cholesterol and triacylglycerol, in part due to the increased expression of genes regulating cholesterol metabolism and lipogenesis. Trail ${ }^{-1}$ Apoe $^{-/}$mice also exhibited severe atherosclerosis. Thus, the regulation of cholesterol and lipogenesis by TRAIL may reduce the severity of diabetic complications and development of atherosclerosis.

\section{Methods}

Animals Trail $^{/-}$mice [11], backcrossed ten times on to C57BL/6 mice [12], were obtained from M. Smyth (Peter MacCallum Cancer Centre, Melbourne, VIC, Australia). $\mathrm{Trail}^{/-}$mice were re-derived and crossed with $\mathrm{Apoe}^{-/-}$ mice (JAX Labs, Bar Harbor, ME, USA) on a C57Bl/6 background. Genotyping for Trail and Apoe was performed as previously described [11] or using JAX Labs protocols for ApoE deficiency. Male mice aged 6 weeks and weighing approximately 18 to $20 \mathrm{~g}$ were placed on a high-fat 'Western' diet (Semi-Pure Rodent Diet SF00-219; $22 \%$ fat, $0.15 \%$ cholesterol; Specialty Feeds, Glen Forrest, WA, Australia) for 12 weeks. Mice were killed by cardiac exsanguination. The aortic root was cannulated and flushed with PBS ( $\mathrm{pH}$ 7.4), followed by perfusion-fixation with $10 \%$ formalin (wt./vol). The aortic tree was removed and immersed in fresh fixative. To evaluate aortic lesions en face, fixed descending aortas were opened longitudinally, pinned out and stained with Oil Red O. Prior to killing, mice were monitored daily and body weights recorded weekly. Food intake was monitored over 4 weeks by placing pre-weighed food of known content into clean cages, under the same conditions. The unconsumed feed was carefully collected, weighed once a week and subtracted from the amount given. Daily intake was calculated across the number of animals housed per cage (two to five), where equal intake of food was assumed. All animal work was conducted according to Animal Care and Ethics Committee guidelines, UNSW (Sydney, NSW, Australia).

Plasma and liver analysis Blood was taken via the tail vein or by cardiac puncture at time of killing. Plasma samples were stored at $-80^{\circ} \mathrm{C}$ in EDTA- $\mathrm{Na}_{2}$ until required for analysis. Triacylglycerol and total cholesterol from plasma (and liver after lipid extraction according to Bligh and Dyer [13]) were measured using commercial kits (Wako Chemicals, Osaka, Japan). Fasting blood glucose and insulin were measured using kits and as described by the manufacturers (Cayman Chemicals, Ann Arbor, MI, USA, and SPI-bio, Ann Arbor, MI, USA, respectively). Lipoprotein analysis on pooled plasma samples was done by fast protein liquid chromatography as previously described [14], using a Superose 6HR 10/30 column (GE Healthcare, Uppsala, Sweden), with $500 \mu$ fractions collected and subjected to cholesterol analysis. Markers of systemic inflammation (monocyte chemoattractant protein-1 [MCP-1], IL-6 and IL-1 $\beta$ ) were assayed using kits (R\&D, Minneapolis, $\mathrm{MN}, \mathrm{USA})$.

FACS analysis Spleens were teased apart and pushed through a $70 \mu \mathrm{m}$ cell strainer (BD Biosciences, Sydney, NSW, Australia). Erythrocytes were lysed in TRIS-buffered ammonium chloride. The remaining cells were washed three times in PBS. Cells were then blocked with PBS containing 5\% heat-inactivated normal rabbit serum, $0.5 \%$ BSA and $2 \mathrm{mmol} / 1 \mathrm{NaN}_{3}$. Subsequently cells were washed with PBS containing $1 \% \mathrm{FCS}$ and $2 \mathrm{mmol} / 1 \mathrm{NaN}_{3}$, then incubated with the following fluorescently conjugated 
antibodies: CD3 (Milteyni Biotec, Sydney, NSW, Australia); CD11c (ebiosciences, San Diego, CA, USA); CD45, $\mathrm{CD} 11 \mathrm{~b}$ and major histocompatibility complex (MHC)-II (BD Pharmingen, Sydney, NSW, Australia). Cells were fixed with $1 \%$ paraformaldehyde (wt./vol). Flow cytometry was performed (FACSCalibur Flow Cytometer; BD Biosciences) and analysed using FCS Express (De Novo Software, Los Angeles, CA, USA).

The HOMA of beta cell function The HOMA of beta cell function (HOMA-B) was used to assess beta cell function. Fasting glucose and insulin were measured at 12 weeks using the HOMA-B equation previously described [15].

Insulin and glucose tolerance tests At 10 weeks of high-fat feeding, either $1 \mathrm{~g} / \mathrm{kg}$ body weight D-glucose (Sigma-Aldrich, Sydney, NSW, Australia) or $1 \mathrm{U} / \mathrm{kg}$ body weight human insulin was injected into mice intraperitoneally following overnight fasting. Blood was collected by pin prick from a tail vein and plasma glucose was measured using a glucometer (Accu-check Performa; Roche, Mannheim, Germany).

$R N A$ extraction and real-time quantitative PCR Liver and intestine were snap-frozen in liquid nitrogen and stored at $-80^{\circ} \mathrm{C}$. Tissue was homogenised (MP Biomedicals, Sydney, NSW, Australia) and total RNA extracted in TRI reagent (Sigma) [1]. RNA was then reverse-transcribed to cDNA using iSCRIPT (Bio-Rad, Sydney, NSW, Australia). Realtime PCR was performed in quadruplicate using Sybr Green Master Mix (Applied Biosystems, Mulgrave, VIC, Australia) in the real-time Corbett 6000 thermocycler (Corbett Life Science, Sydney, NSW, Australia). Relative changes in mRNA levels between groups were determined using the $2^{-\Delta \Delta \mathrm{C}_{\mathrm{t}}}$ method [16]. Expression was normalised to $\beta$-actin and changes were compared with Trail $^{/-}$Apoe $^{-/-}$mice. See electronic supplementary material (ESM) Table 1 for details of the primer sequences.

Histology and immunohistochemistry Brachiocephalic arteries and aortic roots were processed as described [17]. Pancreases were removed and fixed in $10 \%$ formalin (wt./vol). Haematoxylin and eosin stain was used to assess tissue architecture. Arteries were stained for elastin van Gieson, Masson's Trichrome, smooth muscle- $\alpha$ actin (SMA) (1:200; Dako, Sydney, NSW, Australia), macrophages (MAC3) (1:10; BD Pharmingen), active caspase-3 (1:200; R\&D Systems) and proliferating cell nuclear antigen (PCNA; 1:100; Cell Signaling, Danvers, MA USA). Pancreases were stained for insulin (1:500; Cell Signaling), MAC3 (1:100; BD Pharmingen) and active-caspase 3 (1:400; R\&D Systems). All IgG controls were negative. Digital images of sections were acquired using an Olympus DP70 microscope (Olympus, Mount Waverley, VIC, Australia).
Morphometric analysis Morphometric analysis of plaque area:total artery area, media area:total artery area, fibrous cap thickness:plaque area (defined as the VSMC-rich area overlying the necrotic core), necrotic core area:plaque area was performed on elastin-, SMA-, Masson's Trichrome-, and haematoxylin and eosin-stained sections using ImageJ (a public domain Java image processing program inspired by NIH Image) and at least one to two sister sections per artery per mouse. The percentage of positive staining in the plaque or islets was determined using ImageProPlus (Cybernetics, Bethesda, MD, USA). Thresholds for positive staining for each antibody were determined and sections analysed by an investigator blinded to mouse genotype. NIH ImageJ was used to measure area of adipocytes, with at least 100 adipocytes per mouse measured.

Statistics All results are expressed as the mean \pm SEM. Statistical comparisons were made by Mann-Whitney $U$ tests and one- or two-way ANOVA, with Bonferroni's correction where appropriate. The statistics program in GraphPad Prism Version 4.0 was used (GraphPad Software, San Diego, CA, USA). A value of $p<0.05$ was considered significant.

\section{Results}

Trail $^{-}$Apoe $e^{-/-}$mice have increased body weight, consume more food and display adipocyte hypertrophy Compared with Apoe $^{-/-}$mice, Trail ${ }^{-/}$Apoe $e^{-/-}$had significantly increased $(p<0.001)$ body weight at 7 weeks (Fig. 1a), which continued to increase up to 13 weeks. Marked differences in body weight between Trail $^{-1} \mathrm{Apoe}^{-/-}$and Trail $^{-}$mice were observed from 9 weeks of high-fat feeding $(p<0.001)$ (Fig. 1a). Interestingly, Trail $^{-/}$Apoe $^{-/-}$ mice consumed significantly more food daily than the parental strains (Fig. 1b). Significant increases in adipocyte size in white adipose tissue from Trail $^{-1}$ Apoe $^{-/-}$and Trail $^{-}$fat pads were found; adipocytes from the former were consistently larger than those in Apoe $^{-/-}$or Trail $^{/-}$ mice (Fig. 1c). The lack of change in food consumption between Apoe $e^{-/}$and Trail $^{-}$mice suggests that food intake alone did not account for the increased adipocyte hypertrophy observed in $\mathrm{Trai}^{/-}$and $\mathrm{Trail}^{-/} \mathrm{Apoe}^{-/-}$mice.

Trail $^{-}$Apoe $^{-/-}$and Trail ${ }^{--}$mice exhibit altered glucose metabolism To determine potential differences in glucose metabolism, plasma glucose and insulin levels were compared. After 12 weeks on a 'Western' diet, fasting glucose was significantly greater in $\mathrm{Trail}^{-/} \mathrm{Apoe}^{-/-}$than in Apoe $e^{-/-}$and Trail $^{-1-}$ mice (Fig. 2a), whereas plasma insulin levels in $\mathrm{Trail}^{/-}$and $\mathrm{Trail}^{-/}$Apoe $^{-/}$were dramatically reduced (approximately fourfold) (Fig. 2b). Consistent with this, glucose tolerance was impaired in Trail $^{-1}$ Apoe $^{-/-}$and 

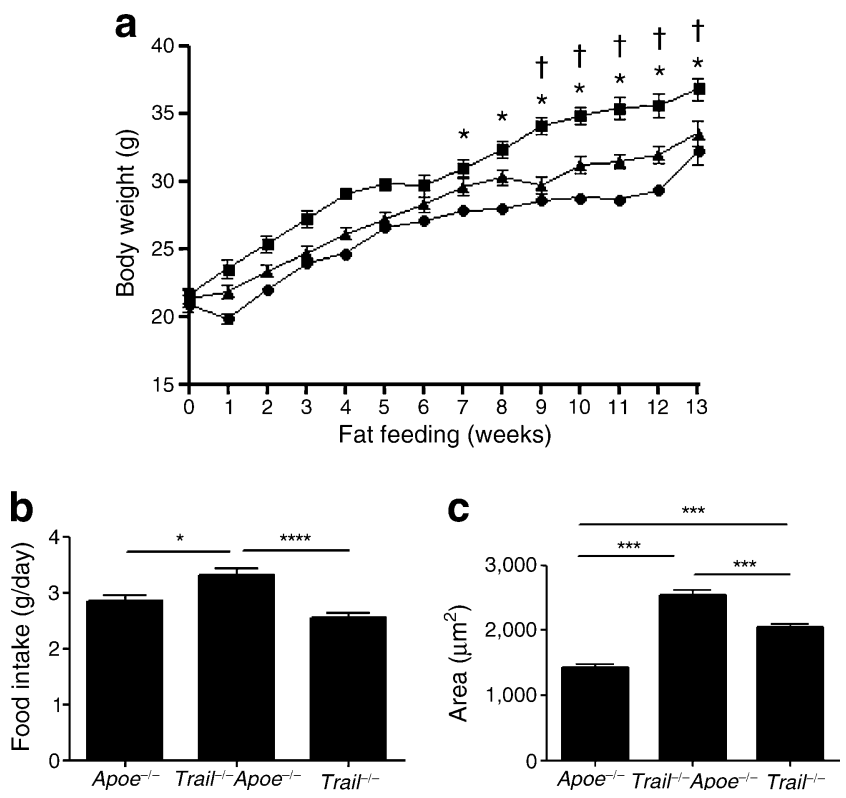

Fig. $1 \mathrm{Trail}^{/-} \mathrm{Apoe}^{-/-}$have increased body weight, consume more food and display adipocyte hypertrophy. a Body weight of Apoe $e^{-/}$ (black circles), Trail $^{\prime-}$ (triangles) and Trail $^{\prime-} \mathrm{Apoe}^{-/-}$(squares) mice aged 6 weeks and fed a high-fat diet for 12 weeks; $n=10-16$ per genotype; ${ }^{*} p<0.05$ for Trail $^{/-}$Apoe $^{-/-}$vs Apoe $^{-/}$; ${ }^{\dagger} p<0.05$ for Trail $^{/-}$Apoe $^{-/-}$vs Trail $^{\prime-}$. b Food intake (g/day); $n=2-5$ per cage per genotype. c $\mathrm{Trail}^{-/} \mathrm{Apoe}^{-/-}$mice display adipocyte hypertrophy compared with Apoe $^{-/-}$and $\mathrm{Trail}^{-1}$ at 12 weeks on a high-fat diet. Results are for adipocyte area $\left(\mu \mathrm{m}^{2}\right) ; n=3$ per genotype. Results are expressed as mean \pm SEM; $* p<0.05,{ }^{* * *} p<0.001$ and $* * * * p<0.0001$, ANOVA used in all conditions

Trail $^{/-}$mice following a glucose challenge (Fig. 2c), whereas no differences in insulin tolerance were seen between all strains (not shown). HOMA using plasma glucose and insulin levels to quantify pancreatic beta cell function (HOMA-B) indicated a significant reduction of beta cell function in Trail $^{-/} \mathrm{Apoe}^{-/-}$ and Trail $^{\prime-}$ mice, namely to approximately $25 \%$ of the levels seen in Apoe $e^{-/}$mice $(p<0.001)$ (Fig. 2d). Interestingly, although high-fat feeding for 12 weeks did not alter plasma $\beta$-hydroxybutyrate levels in Trai $^{-}$mice (0 weeks vs 12 weeks), levels in $A p o e^{-/}$mice were significantly elevated $(p<0.01)$ and levels at 12 weeks of high-fat feeding in Trail $^{-}$Apoe $^{-/-}$were $20 \%$ higher than in Apoe $e^{-/}$mice $(p<0.0001)$ (Fig. 2e), indicating ketosis, a further feature of diabetes.

Examination of pancreatic tissue revealed no significant differences in islet diameter or area (not shown), whereas pancreases from Trail $^{-}$Apoe $^{-/}$and Trail $^{-}$mice contained significantly reduced endogenous insulin staining in islets (Fig. 2f). Although MAC3-positive macrophage numbers infiltrating the pancreas were significantly greater than those seen in $A$ poe $e^{-/}$mice, there were approximately 2.5 -fold more MAC3-positive cells in Trail $^{\prime-}$ Apoe $^{-/}$than in Trail $^{-/}$mice pancreases (Fig. 2g). Moreover, Trai ${ }^{-/}$Apoe $e^{-/}$pancreases showed significantly increased apoptosis, as indicated by cleaved caspase-3 staining $(p<0.05)$; this was also seen to a lesser extent in $\mathrm{Trail}^{-/}$compared with $A$ poe $e^{-/-}$islets (Fig. 2h). Together, these findings suggest that $\mathrm{Trail}^{-} \mathrm{Apoe}^{-/-}$and Trail $^{--}$animals have a deficiency in insulin production that is probably due to pancreatic inflammation and apoptosis, and results in increased blood glucose levels.

Splenic leucocytes and plasma IL-6 are increased in Trail $^{/-}$Apoe $^{-/-}$and Trail ${ }^{--}$mice TRAIL plays a critical role in the immune system. To determine mechanisms for the differences observed between strains, we assessed levels of immune cells from spleens using flow cytometry. MHC-II molecules are found on antigen-presenting cells such as dendritic cells and macrophages. MHC-II-expressing cell numbers in Trai $^{-}{ }^{-}$Apoe $e^{-/-}$and Trail $^{\prime-}$ mice were similar, but fewer than in Apoe $e^{-/-}$mice (Table 1). The reduced numbers of CD11c-positive cells suggest that Apoe $e^{-/-}$ spleens contained markedly higher numbers of dendritic cells. In marked contrast, Trail ${ }^{-}$Apoe $^{-/}$and Trail $^{-/}$had a greater number of cells expressing CD11b, a marker for leucocytes (Table 1), and the numbers from Trail $^{-}$mice were significantly higher than those from $\mathrm{Trail}^{/-} \mathrm{Apoe}^{-/-}$ mice $(p<0.001)$ (Table 1). No differences in CD3-expressing cells (T cells) were observed (Table 1). Taken together, these findings indicate a reduced number of dendritic cells, but marked increases in phagocytic cells, probably macrophages, in Trail $^{/-}$Apoe $^{-/-}$and Trail ${ }^{-}$spleens.

Increased circulating IL-6 and MCP-1 are associated with type 2 diabetes [18-20]. A pro-inflammatory cytokine, IL-6 is produced by macrophages in response to infection or tissue insult [21]. Trail $^{/-}$Apoe $^{-/-}$and Trail $^{/-}$mice had significantly elevated plasma IL-6 values, which were highest in Trail $^{-}$animals, possibly reflecting the high levels of CD11b-positive leucocytes (Table 1). MCP-1 is a chemokine that recruits monocytes to areas of injury and is important in atherogenesis [22]. Although no significant differences in plasma MCP-1 were observed between

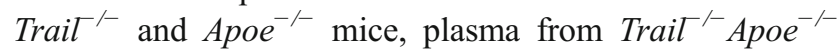
contained significantly higher levels than plasma from Trail $^{\prime-}$ mice $(p<0.05)$ (Table 1$)$. In contrast, plasma IL-1 $\beta$ levels were similar in all strains (Table 1 ).

Trail $^{/-}$Apoe ${ }^{-/-}$mice have increased plasma cholesterol and triacylglycerol As expected [23], plasma cholesterol levels in Apoe $e^{-/-}$mice increased after 12 weeks of high-fat feeding from $6.58 \pm 0.61 \mathrm{mmol} / 1$ to $15.73 \pm 1.39 \mathrm{mmol} / 1$, whereas those in Trail $^{--}$mice (which are Apoe $^{+/+}$) remained at the levels seen at 0 weeks $(2.835 \pm 0.14)$. In contrast, plasma cholesterol levels in $\mathrm{Trail}^{-/} \mathrm{Apoe}^{-/-}$mice were markedly elevated, namely from $9.0 \pm 0.57 \mathrm{mmol} / 1$ to $23.02 \pm 1.34 \mathrm{mmol} / \mathrm{l}$, and significantly higher than levels seen in Apoe $e^{-/-}$mice $(p<0.001)$ (Table 1). Plasma triacylglycerol levels were also significantly greater in Trail $^{-}$Apoe $^{-/-}$mice (Table 1). Importantly, plasma from 

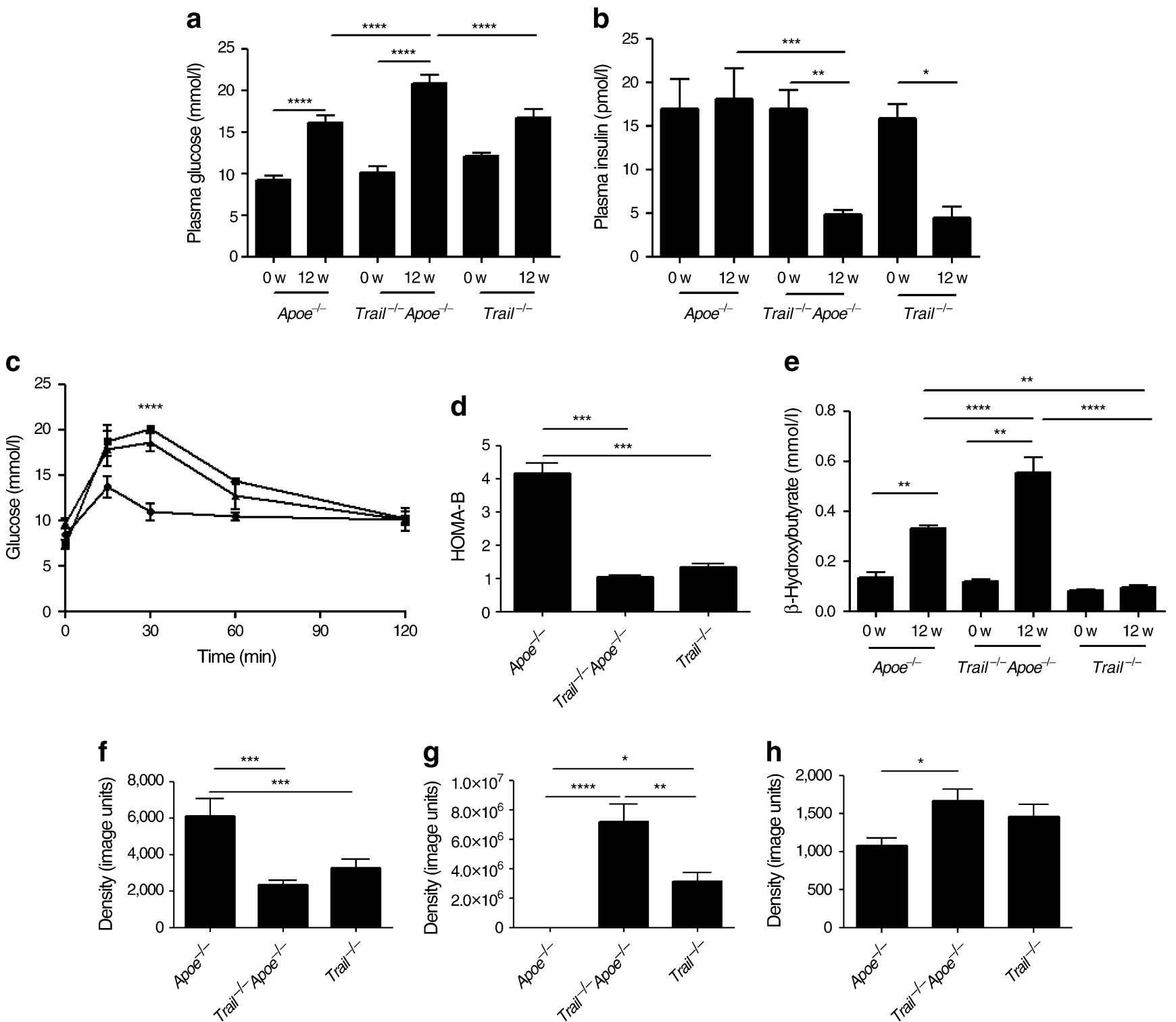

Fig. $2 \mathrm{Trail}^{/-} \mathrm{Apoe}^{-/-}$and $\mathrm{Trail}^{-/}$mice have altered glucose metabolism. a Fasting plasma glucose $(n=10-14)$, (b) fasting plasma insulin ( $n=7-17$ per genotype) and (c) glucose tolerance test for $\mathrm{Apoe}^{-/-}$(circles), $\mathrm{Trail}^{--}$(triangles) and $\mathrm{Trail}^{-/} \mathrm{Apoe}^{-/-}$(squares) mice at week 0 and week 12 ( $n=3-5$ per genotype). d Assessment of HOMA-B as described in Methods; $n=7-11$ per genotype. e Plasma ketone bodies detected using a $\beta$-hydroxybutyrate assay; $n=4-5$ per genotype. f Mouse pancreatic islets stained for insulin, (g) macrophages (MAC3) and (h) apoptosis (active caspase-3); $n=5-7$ per genotype. Results are expressed as mean $\pm \mathrm{SEM} ;{ }^{*} p<0.05,{ }^{* *} p<0.01,{ }^{* * *} p<0.001$ and $* * * * p<0.0001$, ANOVA used in all conditions. w, weeks

mately threefold higher cholesterol and triacylglycerol levels than $A$ poe $e^{-/-}$mice, and approximately 12-fold (triacylglycerol) and 40-fold (cholesterol) higher levels than Trail $^{/-}$mice (Fig. 3a, b). To determine the mechanisms underlying this hypercholesterolaemia and hypertriacylglycerolaemia, we examined the expression of genes involved in cholesterol metabolism. 3-Hydroxy-3-methyl-glutarylCoA reductase (HMGCoAR), the rate-limiting enzyme in the cholesterol metabolic pathway, is rapidly induced in mice by high-fat feeding [24]. Hmgcoar mRNA expression was significantly elevated in $\mathrm{Trail}^{-1} \mathrm{Apoe}^{-/-}$mice (Fig. 3c). (Table 1), livers from $\mathrm{Trail}^{-/} \mathrm{Apoe}^{-/-}$mice had approxi- 
Table 1 Plasma chemistry and inflammatory markers

\begin{tabular}{|c|c|c|c|}
\hline & Apoe $e^{-/}$ & Trail $^{/-}$Apoe $^{-/-}$ & Trail $^{/-}$ \\
\hline Cholesterol (mmol/l) & $15.73 \pm 1.39$ & $23.02 \pm 1.36^{* \dagger}$ & $3.61 \pm 0.46$ \\
\hline Triacylglycerol (mmol/l) & $1.10 \pm 0.07$ & $2.21 \pm 0.14^{* \dagger}$ & $0.65 \pm 0.02$ \\
\hline VLDL-cholesterol (mmol/l) & $0.59 \pm 0.04$ & $0.71 \pm 0.03^{* \dagger}$ & $0.03 \pm 0.003$ \\
\hline LDL-cholesterol (mmol/l) & $0.11 \pm 0.09$ & $0.23 \pm 0.11^{* \dagger}$ & $0.08 \pm 0.003$ \\
\hline HDL-cholesterol (mmol/1) & - & $0.06 \pm 0.03^{\dagger}$ & $0.18 \pm 0.007$ \\
\hline IL-6 (pg/ml) & $9.92 \pm 2.53$ & $23.07 \pm 3.68^{*}$ & $36.68 \pm 5.92$ \\
\hline MCP-1 (pg/ml) & $86.23 \pm 2.49$ & $128 \pm 20.24^{\dagger}$ & $72.62 \pm 9.13$ \\
\hline IL-1 $\beta(\mathrm{pg} / \mathrm{ml})$ & $7.22 \pm 2.04$ & $10.25 \pm 2.7$ & $11.38 \pm 1.09$ \\
\hline MHC-II & $69.53 \pm 2.20$ & $57.43 \pm 3.24^{*}$ & $48.07 \pm 1.43$ \\
\hline CD11c & $19.63 \pm 1.34$ & $6.47 \pm 0.65^{*}$ & $6.26 \pm 0.42$ \\
\hline CD11b & $10.92 \pm 0.39$ & $85.37 \pm 1.37^{*}$ & $93.00 \pm 0.42$ \\
\hline CD3 & $35.89 \pm 1.29$ & $38.53 \pm 1.16$ & $42.80 \pm 1.90$ \\
\hline
\end{tabular}

Results are expressed as mean \pm SEM

Fasting plasma cholesterol, $n=7-8$ per genotype; fasting plasma triacylglycerol, $n=6-8$ per genotype

Lipoprotein profiles (VLDL-, LDL-, HDL-cholesterol) were analysed by fast protein liquid chromatography and cholesterol levels determined, $n=6$ per genotype

Plasma IL-6 ( $n=5-8$ per genotype), MCP-1 ( $n=5-6$ per genotype) and IL-1 $\beta$ ( $n=5-11$ per genotype) were measured using commercial assays Mouse spleen cells were analysed for MHC-II, CD11, CD11b and CD3 by flow cytometry, $n=3-7$ per genotype

${ }^{*} p<0.05$ for Trail $^{/-}$Apoe $^{-/-}$vs Apoe ${ }^{-/-} ;{ }^{\dagger} p<0.05$ for Trail $^{/-}$Apoe $^{-/-}$vs Trail $^{-/}$; ANOVA was used in all conditions

Neimann-Pick C1-like 1 is a sterol transporter of cholesterol for intestinal absorption [25]. Expression of Npc1l1 was also significantly elevated in Trail $^{\prime-}$ Apoe $e^{-/}$mice (Fig. 3d), whereas Abcg5 mRNA, a gene important for cholesterol transport, was similar in all strains (Fig. 3e). Thus, increased cholesterol synthesis and absorption are likely to contribute to the elevated cholesterol levels seen in $\mathrm{Trail}^{-\alpha} \mathrm{Apoe}^{-/}$mice.

To determine whether increased lipogenesis may play a role in the elevated production of triacylglycerol in the liver, and in the increases of plasma VLDL- and LDLcholesterol observed in $\mathrm{Trail}^{/-} \mathrm{Apoe}^{-/-}$mice, we analysed mRNA levels of key lipogenic genes. Expression of Srebp 1, Glut 2 and Ldlr was significantly higher for all three genes in livers from Trail $^{/-}$Apoe $^{-/-}$compared with Apoe $^{-/}$or Trail ${ }^{/-}$mice (Fig. 3f-h). Thus, in an Apoe $e^{-/-}$ background, TRAIL-deficiency promotes elevated expression of genes that regulate cholesterol synthesis and absorption, and triacylglycerol production.

Trail $^{-1}$ Apoe $^{-1-}$ mice have increased atherosclerosis $\mathrm{Trail}^{\prime-} \mathrm{Apoe}^{-/-}$mice showed dramatically increased aortic atherosclerosis (Fig. 4a). Their brachiocephalic arteries had significantly greater plaque area and medial expansion than those of Apoe $e^{-/}$mice (Fig. 4b-d), and aortic root atherosclerosis was also increased (Fig. 4e, f). In marked contrast, arteries from $\mathrm{Trai}^{\prime-}$ mice appeared healthy with no signs of atherosclerosis (Fig. 4b). Interestingly, brachiocephalic plaques from Trail $^{-1}$ Apoe $^{-/}$had significantly less SMA staining, indicating reduced VSMC content (Fig. 5a; Table 2). Collagen content (Masson's Trichrome) (Fig. 5b; Table 2) and cap thickness were also significantly reduced in $\mathrm{Trail}^{-/} \mathrm{Apoe}^{-/-}$compared with Apoe $^{-/-}$plaques (Table 2). Trail $^{/-}$Apoe $e^{-/-}$plaques had more extensive necrotic cores and macrophage infiltration, with almost double the infiltration in $A p o e^{-/}$samples (Fig. 5c; Table 2). Although PCNA (cell proliferation) levels were not changed (Fig. 5d; Table 2), significantly increased caspase- 3 immunoreactivity was observed in Trai $^{/-}$Apoe $^{-/-}$compared with Apoe $e^{-/-}$plaques, particularly in the shoulder regions (Fig. 5e; Table 2).

\section{Discussion}

In humans with CVD, soluble TRAIL levels are reduced [9] and this has been linked with increased risk of cardiovascular events, independently of conventional risk markers [26]. Reduced circulating TRAIL in older patients with CVD is also associated with increased risk of death over a 6 year period [27]. Furthermore, patients with myocardial infarction display reduced TRAIL levels compared with healthy controls [26], implicating a protective role for TRAIL in these clinical conditions.

TRAIL is a cell surface and soluble ligand that activates TRAIL receptors, of which there are five in humans [2]. TRAIL plays an important role in homeostasis of the immune system, as well as in infection, autoimmune and cancer diseases; it also has apoptotic, 

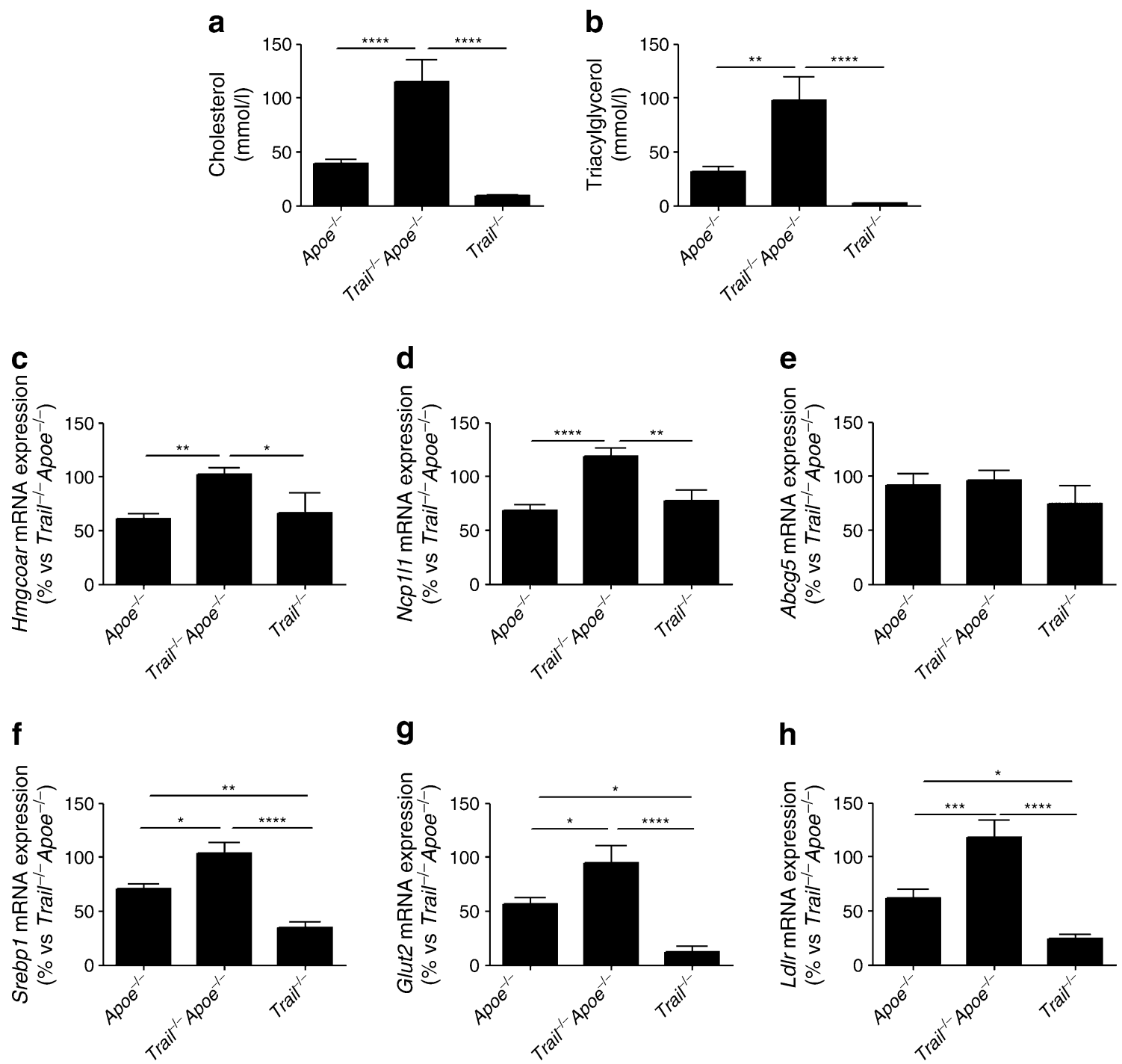

Fig. $3 \mathrm{Trail}^{/-} \mathrm{Apoe}^{-/-}$mice have altered expression of genes regulating cholesterol metabolism and lipogenesis. a Hepatic cholesterol and (b) triacylglycerol content was determined after lipid extraction; $n=4-5$ per genotype. c Hmgcoar mRNA expression was detected from liver; (d) Npc1l1 mRNA was detected from intestine; (e) Abcg 5 mRNA levels were detected from the liver. f Srebp1, (g) Glut2 and (h) Ldlr mRNA were measured from liver. All mRNA levels were normalised to $\beta$-actin; $n=6-8$ per genotype. Results are expressed as mean \pm SEM; ${ }^{*} p<0.05, * * p<0.01 * * * p<0.001$ and $* * * * p$ $<0.0001$, as assessed by ANOVA

sis, a major diabetic complication; these conditions are probably intensified by the chronic inflammation observed in $\mathrm{Trail}^{-1} \mathrm{Apoe}^{-/-}$mice.

Type 2 diabetic patients have increased levels of LDLcholesterol and triacylglycerol, and lower HDL-cholesterol than non-diabetic patients [29], and are more frequently overweight [30]. Although no correlation between circulating TRAIL and BMI was found in patients with CVD [31], a positive correlation between soluble TRAIL, serum LDLcholesterol and total body fat in elderly men and women has been reported [32]. We found that Trail ${ }^{\prime-} \mathrm{Apoe}^{-/}$mice ate more, were significantly heavier and displayed adipocyte hypertrophy compared with parental strains. Compared with Apoe $^{-/}$, Trail $^{--}$mice had increased adiposity, suggesting 
a

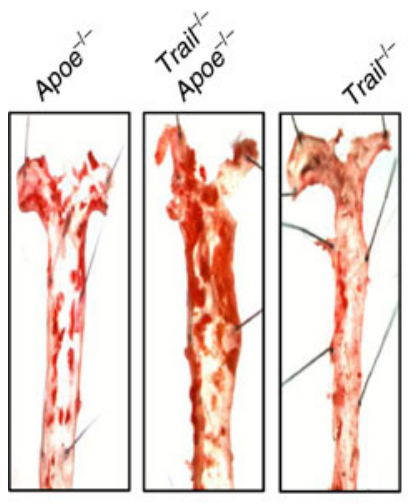

b

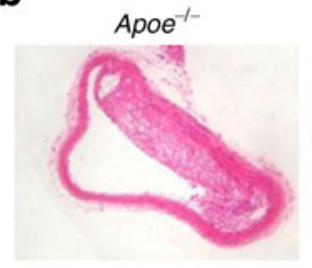

C

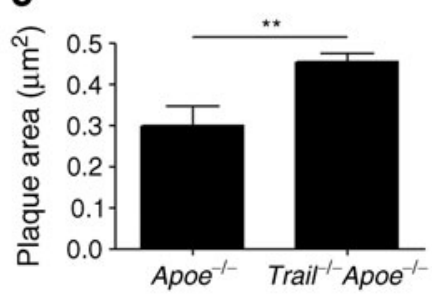

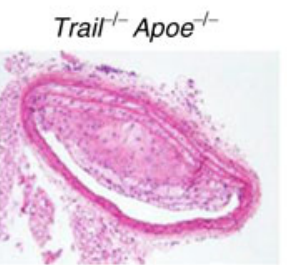
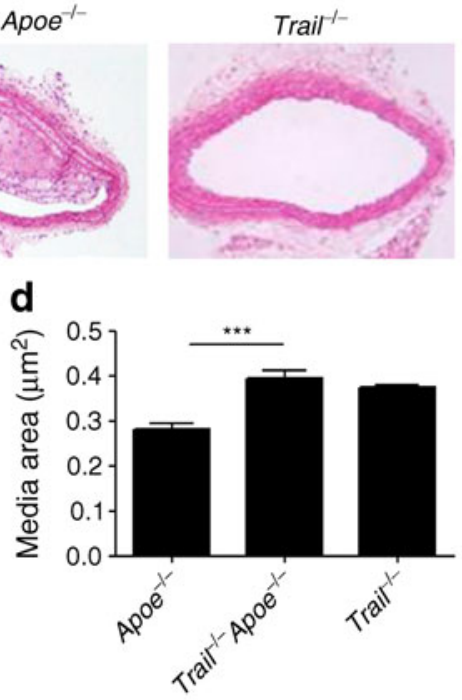

f

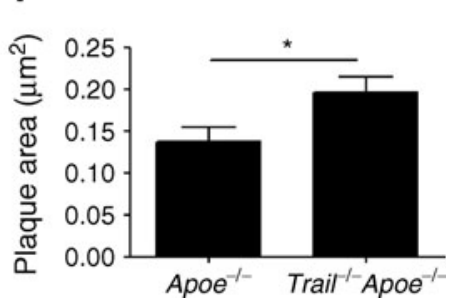

Apoe Trail $^{-1-}$ Apoe $^{-1-}$ e

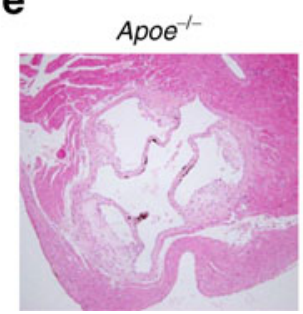

Trail $^{-1-}$ Apoe $^{-1-}$

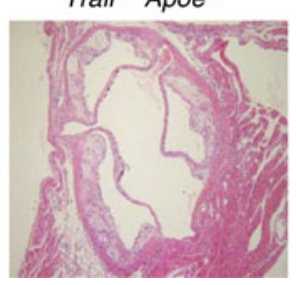

Trail $^{1-}$

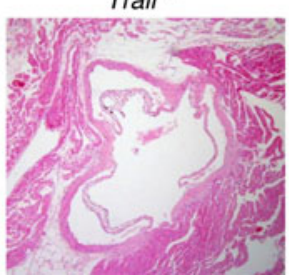

Fig. 4 Trail $^{-}$Apoe $^{-/-}$have increased atherosclerosis. a En face aortic staining for Oil Red O (magnification $\times 2.5$ ). b Representative haematoxylin and eosin-stained cross sections (magnification $\times 20$ ) of brachiocephalic arteries of mice as indicated at 12 weeks of high-fat diet. c Plaque and (d) medial area measurements were performed as described in the Methods; $n=8-12$ per genotype. e Representative haematoxylin and eosin-stained cross sections (magnification $\times 10$ ) of aortic sinus of mice as indicated at 12 weeks of high-fat diet. $\mathbf{f}$ Plaque area measurements were performed as described in the Methods; $n=8-12$ per genotype. Results are expressed as mean \pm SEM; ${ }^{*} p<0.05,{ }^{* *} p<0.01$ and ${ }^{* * *} p<0.001$ by Mann-Whitney $U$ test and ANOVA

an approximately eightfold higher number of CD11bpositive splenic leucocytes, as well as increases in the inflammatory marker IL-6. The plasma IL-6 levels observed in Trail $^{/-}$Apoe $^{-/-}$mice may be a gene-dose effect, since levels reflect an intermediate concentration between Apoe $^{-/-}$and $\mathrm{Trail}^{-/}$. Rather than playing a role in atherosclerosis (Trail $^{--}$mice do not develop lesions), IL-6 is more likely to affect the pancreas. Interestingly, IL-6 secreted by inflammatory cells can induce beta cell death resulting in reduced insulin production [35]. In contrast, levels of plasma MCP-1 were more elevated in $\mathrm{Trail}^{/-} \mathrm{Apoe}^{-/}$and may contribute to the increased numbers of macrophages seen in pancreases and in atherosclerotic plaques (Fig. 2g and 5c, Table 2). In both cases, macrophage-dense areas were approximately double those seen in Apoe $e^{-/}$mice (Fig. $2 \mathrm{~g}$ and $5 \mathrm{c}$, Table 2). TRAIL may be involved in monocyte recruitment to sites of chronic inflammation, as seen in type 1 diabetes [36] and in atherosclerosis [37].

Lack of TRAIL in Apoe $e^{-/}$mice caused altered lipid metabolism, with significantly increased plasma cholesterol and triacylglycerol. There are three mechanisms to account for 
a

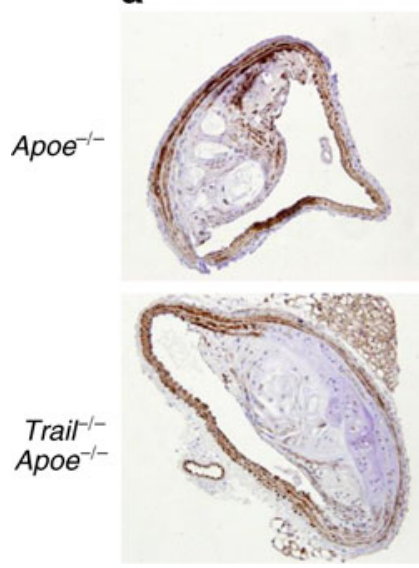

b

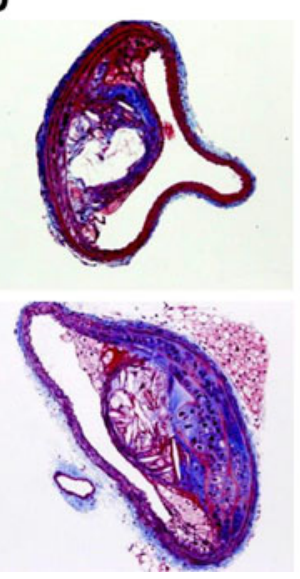

C

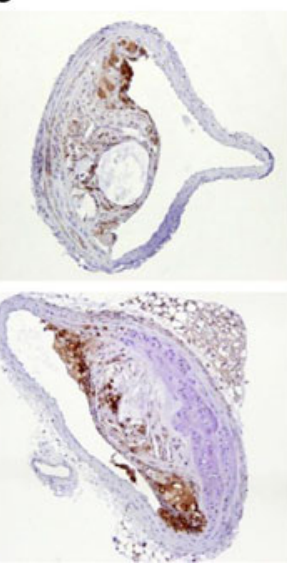

d

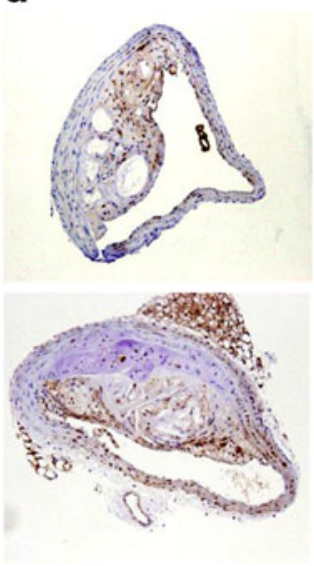

e

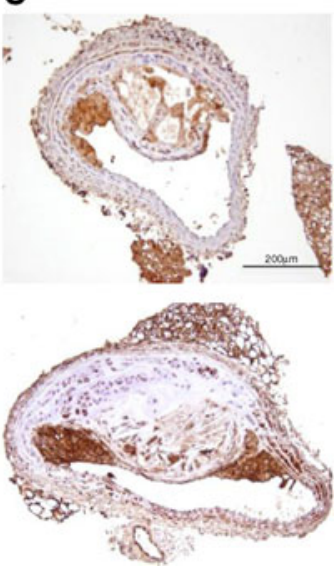

Fig. $5 \mathrm{Trail}^{-1} \mathrm{Apoe}^{-/}$mice have altered plaque cellular composition. Representative cross sections (magnification $\times 20$ ) of brachiocephalic mouse plaques stained for (a) VSMCs (SMA), (b) collagen (Masson's
Trichrome), (c) macrophages (MAC3), (d) cell proliferation (PCNA) and (e) apoptosis (active caspase-3)

Trail $^{-1}$ Apoe $^{-/-}$mice displayed signs of accelerated atherosclerosis and features of plaque vulnerability. This includes thinning of the fibrous cap, decreased VSMC and collagen content, elevated macrophage accumulation, outward plaque remodelling and increased necrotic core size [44]. These findings implicate TRAIL in the maintenance of plaque integrity. We showed increased apoptosis in the vulnerable regions of plaques, where macrophage infiltration was also evident. Although our results are consistent with the observation that systemic TRAIL administration can attenuate plaque development in vivo in diabetic Apoe $e^{-/}$mice [7], they are not consistent with a recent study also implicating TRAIL in the attenuation of atherosclerosis [45]. At 12 weeks on a 'Western' diet, Watt et al. found no differences in cholesterol or glucose levels between $\mathrm{Trail}^{-/} \mathrm{Apoe}^{-/-}$and Apoe animals [45], although insulin levels were not checked and tolerance testing was not performed. Moreover, contents of 'Western' diets vary and the composition of that used by Watt et al. was not specified. We believe the differences in lesion development and plasma chemistry seen in these studies may result from differences in the fat content of the diets.

It is clear from these studies that hyperglycaemia alone is insufficient to accelerate atherosclerosis in this model, since no lesions were apparent in Trail $^{-/}$mice. However, in our

Table 2 Quantification of plaque cap thickness, necrotic core and immunostains

\begin{tabular}{|c|c|c|c|c|c|c|c|}
\hline & $\begin{array}{l}\text { Plaque cap } \\
\text { thickness (\%) }\end{array}$ & $\begin{array}{l}\text { Necrotic } \\
\text { core }(\%)\end{array}$ & $\begin{array}{l}\text { Caspase-3 } \\
\text { (image units) }\end{array}$ & $\begin{array}{l}\text { SMA } \\
\text { (image units) }\end{array}$ & $\begin{array}{l}\text { MAC3 } \\
\text { (image units) }\end{array}$ & $\begin{array}{l}\text { Masson's Trichrome } \\
\text { (image units) }\end{array}$ & $\begin{array}{l}\text { PCNA } \\
\text { (image units) }\end{array}$ \\
\hline Apoe $e^{-1-}$ & $0.15 \pm 0.016$ & $21.69 \pm 3.59$ & $46.84 \pm 2.44$ & $34.37 \pm 5.99$ & $5.55 \pm 1.53$ & $44.0 \pm 11.22$ & $5.64 \pm 0.8$ \\
\hline Trail $^{/-}$Apoe $^{-/-}$ & $0.076 \pm 0.014^{*}$ & $39.30 \pm 5.46^{*}$ & $70.57 \pm 5.35^{*}$ & $15.72 \pm 2.17^{*}$ & $11.27 \pm 2.19^{*}$ & $16.57 \pm 3.39^{*}$ & $4.92 \pm 0.58$ \\
\hline
\end{tabular}

Plaque cap thickness and necrotic core were quantified using brachiocephalic arteries ( $n=7-8$ per genotype) as described in Methods ImageProPlus was used to quantify SMA, Masson's Trichrome, macrophages (MAC3), PCNA and active caspase-3 as described in Methods. ${ }^{*} p<0.05$ or greater, Trail $\Gamma^{/-}$Apoe $e^{-/-}$vs Apoe ${ }^{-/-}$; Mann-Whitney $U$ test 
system, hyperglycaemia in concert with hyperlipidaemia leads to accelerated atherosclerosis. This is not surprising, as glucose and lipids act by the same mechanisms to initiate atherosclerotic lesions [46]. Increased infiltrating macrophages in $\mathrm{Trail}^{-1} \mathrm{Apoe}^{-/-}$mice may stimulate movement of monocytes/macrophages or systemic inflammatory chemokines (such as MCP-1) to lesions and contribute to the severity of atherosclerosis. S100A9 is a biomarker for CVD. Interestingly, in a mouse model of diabetes, increased accumulation of S100A9-positive monocytic cells was observed in lesions [47], supporting the notion that an inflammatory state contributes to atherosclerotic lesions in diabetes. In our model, diabetes was not artificially induced and atherosclerosis in $A p o e^{-/}$mice was exacerbated by the lack of TRAIL. We confirm a significant link between TRAIL, diabetes and atherosclerosis, and prove a causative rather than a consequential role for TRAIL in these pathological complications. More detailed comparisons of Trail $^{-1} \mathrm{Apoe}^{-/-}$and the parental mouse strains should further our understanding of TRAIL-dependent cellular/ molecular mechanisms of diabetes and its complications.

Acknowledgements Trai $^{-/}$mice were kindly provided by M. Smyth and originally sourced from AMGEN (AMGEN Extramural Research Alliances, Seattle, WA, USA). We would like to thank W. Jessup (Centre for Vascular Research, University of New South Wales, Sydney, Australia) for discussions on lipid metabolism and C. Geczy (School of Medical Sciences, University of New South Wales, Sydney, Australia) for critical review of the manuscript. This work was supported by National Health and Medical Research Council (NHMRC) project grant 568627. M.M. Kavurma is supported by a University of New South Wales Vice-Chancellor's Fellowship. M.R. Bennett is supported by the British Heart Foundation and the National Institute for Health Research Cambridge Biomedical Research Centre.

Contribution statement $\mathrm{BAD}$ and $\mathrm{MMK}$ were responsible for experimentation, conception, design, analysis and interpretation of data, manuscript preparation and intellectual input, and gave final approval for publication. JC and SC were responsible for experimentation, interpretation of data, manuscript preparation and intellectual input, and gave final approval for publication. MRB was responsible for interpretation of data, manuscript preparation and intellectual input, and gave final approval for publication. SB and BET were responsible for analysis and interpretation of data, manuscript preparation and intellectual input, and gave final approval for publication.

Duality of interest The authors declare that there is no duality of interest associated with this manuscript.

\section{References}

1. Chan J, Prado-Lourenco L, Khachigian LM, Bennett MR, Di Bartolo BA, Kavurma MM (2010) TRAIL promotes VSMC proliferation and neointima formation in a FGF-2-, Sp1 phosphorylation-, and NFkappaB-dependent manner. Circ Res 106:1061-1071

2. Kavurma MM, Bennett MR (2008) Expression, regulation and function of trail in atherosclerosis. Biochem Pharmacol 75:14411450
3. Kavurma MM, Schoppet M, Bobryshev YV, Khachigian LM, Bennett MR (2008) Trail stimulates proliferation of vascular smooth muscle cells via activation of NF-kappa B and induction of insulinlike growth factor-1 receptor. J Biol Chem 283:7754-7762

4. Kavurma MM, Tan NY, Bennett MR (2008) Death receptors and their ligands in atherosclerosis. Arterioscler Thromb Vasc Biol 28:1694-1702

5. Lamhamedi-Cherradi SE, Zheng S, Tisch RM, Chen YH (2003) Critical roles of tumor necrosis factor-related apoptosis-inducing ligand in type 1 diabetes. Diabetes 52:2274-2278

6. Lamhamedi-Cherradi SE, Zheng SJ, Maguschak KA, Peschon J, Chen YH (2003) Defective thymocyte apoptosis and accelerated autoimmune diseases in $\mathrm{TRAIL}^{-1-}$ mice. Nat Immunol 4:255-260

7. Secchiero P, Candido R, Corallini F et al (2006) Systemic tumor necrosis factor-related apoptosis-inducing ligand delivery shows antiatherosclerotic activity in apolipoprotein E-null diabetic mice. Circulation 114:1522-1530

8. Corallini F, Celeghini C, Rizzardi C et al (2007) Insulin downregulates TRAIL expression in vascular smooth muscle cells both in vivo and in vitro. J Cell Physiol 212:89-95

9. Schoppet M, Sattler AM, Schaefer JR, Hofbauer LC (2006) Osteoprotegerin (OPG) and tumor necrosis factor-related apoptosis-inducing ligand (TRAIL) levels in atherosclerosis. Atherosclerosis 184:446-447

10. Zhang SH, Reddick RL, Piedrahita JA, Maeda N (1992) Spontaneous hypercholesterolemia and arterial lesions in mice lacking apolipoprotein E. Science 258:468-471

11. Sedger LM, Glaccum MB, Schuh JC et al (2002) Characterization of the in vivo function of TNF-alpha-related apoptosis-inducing ligand, TRAIL/Apo2L, using TRAIL/Apo2L gene-deficient mice. Eur J Immunol 32:2246-2254

12. Cretney E, Takeda K, Yagita H, Glaccum M, Peschon JJ, Smyth MJ (2002) Increased susceptibility to tumor initiation and metastasis in TNF-related apoptosis-inducing ligand-deficient mice. J Immunol 168:1356-1361

13. Bligh EG, Dyer WJ (1959) A rapid method of total lipid extraction and purification. Can J Biochem Physiol 37:911-917

14. Garber DW, Kulkarni KR, Anantharamaiah GM (2000) A sensitive and convenient method for lipoprotein profile analysis of individual mouse plasma samples. J Lipid Res 41:1020-1026

15. Hermans MP, Levy JC, Morris RJ, Turner RC (1999) Comparison of tests of beta-cell function across a range of glucose tolerance from normal to diabetes. Diabetes 48:1779-1786

16. Bustin SA (2000) Absolute quantification of mRNA using realtime reverse transcription polymerase chain reaction assays. J Mol Endocrinol 25:169-193

17. Clarke MC, Figg N, Maguire JJ et al (2006) Apoptosis of vascular smooth muscle cells induces features of plaque vulnerability in atherosclerosis. Nat Med 12:1075-1080

18. Pickup JC, Chusney GD, Thomas SM, Burt D (2000) Plasma interleukin-6, tumour necrosis factor alpha and blood cytokine production in type 2 diabetes. Life Sci 67:291-300

19. Piemonti L, Calori G, Lattuada G et al (2009) Association between plasma monocyte chemoattractant protein-1 concentration and cardiovascular disease mortality in middle-aged diabetic and nondiabetic individuals. Diabetes care 32:2105-2110

20. Senn JJ, Klover PJ, Nowak IA, Mooney RA (2002) Interleukin-6 induces cellular insulin resistance in hepatocytes. Diabetes 51:3391-3399

21. Omoigui S (2007) The interleukin-6 inflammation pathway from cholesterol to aging - role of statins, bisphosphonates and plant polyphenols in aging and age-related diseases. Immun Ageing 4:1

22. Aiello RJ, Bourassa PA, Lindsey S et al (1999) Monocyte chemoattractant protein-1 accelerates atherosclerosis in apolipoprotein E-deficient mice. Arterioscler Thromb Vasc Biol 19:15181525 
23. Sehayek E, Shefer S, Nguyen LB, Ono JG, Merkel M, Breslow JL (2000) Apolipoprotein E regulates dietary cholesterol absorption and biliary cholesterol excretion: studies in C57BL/6 apolipoprotein E knockout mice. Proc Natl Acad Sci U S A 97:3433-3437

24. Roberts CK, Liang K, Barnard RJ, Kim CH, Vaziri ND (2004) HMG-CoA reductase, cholesterol 7alpha-hydroxylase, LDL receptor, SR-B1, and ACAT in diet-induced syndrome X. Kidney Int 66:1503-1511

25. Jia L, Betters JL, Yu L (2010) Niemann-pick C1-like 1 (NPC1L1) protein in intestinal and hepatic cholesterol transport. Annu Rev Physiol 73:239-259

26. Secchiero P, Corallini F, Ceconi C et al (2009) Potential prognostic significance of decreased serum levels of TRAIL after acute myocardial infarction. PloS one 4:e4442

27. Volpato S, Ferrucci L, Secchiero P et al (2011) Association of tumor necrosis factor-related apoptosis-inducing ligand with total and cardiovascular mortality in older adults. Atherosclerosis 215:452-458

28. Secchiero P, Corallini F, Pandolfi A et al (2006) An increased osteoprotegerin serum release characterizes the early onset of diabetes mellitus and may contribute to endothelial cell dysfunction. Am J Pathol 169:2236-2244

29. Stout RW (1987) Insulin and atheroma-an update. Lancet 1:1077-1079

30. Garcia MJ, McNamara PM, Gordon T, Kannel WB (1974) Morbidity and mortality in diabetics in the Framingham population. Sixteen year follow-up study. Diabetes 23:105-111

31. Mori K, Ikari Y, Jono S et al (2011) Association of serum TRAIL level with coronary artery disease. Thromb Res 125:322-325

32. Choi JW, Song JS, Pai SH (2004) Associations of serum TRAIL concentrations, anthropometric variables, and serum lipid parameters in healthy adults. Ann Clin Lab Sci 34:400-404

33. Dirice E, Sanlioglu AD, Kahraman S et al (2009) Adenovirusmediated TRAIL gene (Ad5hTRAIL) delivery into pancreatic islets prolongs normoglycemia in streptozotocin-induced diabetic rats. Hum Gene Ther 20:1177-1189

34. Zauli G, Toffoli B, di Iasio MG, Celeghini C, Fabris B, Secchiero P (2010) Treatment with recombinant tumor necrosis factor-related apoptosis-inducing ligand alleviates the severity of streptozotocininduced diabetes. Diabetes 59:1261-1265

35. Robertson RP, Zhang HJ, Pyzdrowski KL, Walseth TF (1992) Preservation of insulin mRNA levels and insulin secretion in HIT cells by avoidance of chronic exposure to high glucose concentrations. J Clin Invest 90:320-325

36. Devaraj S, Glaser N, Griffen S, Wang-Polagruto J, Miguelino E, Jialal I (2006) Increased monocytic activity and biomarkers of inflammation in patients with type 1 diabetes. Diabetes 55:774-779

37. Binder CJ, Chang MK, Shaw PX et al (2002) Innate and acquired immunity in atherogenesis. Nat Med 8:1218-1226

38. Altmann SW, Davis HR Jr, Zhu LJ et al (2004) Niemann-Pick C1 Like 1 protein is critical for intestinal cholesterol absorption. Science 303:1201-1204

39. Davies JP, Scott C, Oishi K, Liapis A, Ioannou YA (2005) Inactivation of NPC1L1 causes multiple lipid transport defects and protects against diet-induced hypercholesterolemia. J Biol Chem 280:12710-12720

40. Marks J, Carvou NJ, Debnam ES, Srai SK, Unwin RJ (2003) Diabetes increases facilitative glucose uptake and GLUT2 expression at the rat proximal tubule brush border membrane. J Physiol 553:137-145

41. Rencurel F, Waeber G, Antoine B et al (1996) Requirement of glucose metabolism for regulation of glucose transporter type 2 (GLUT2) gene expression in liver. Biochem J 314:903-909

42. Im SS, Kang SY, Kim SY et al (2005) Glucose-stimulated upregulation of GLUT2 gene is mediated by sterol response element-binding protein-1c in the hepatocytes. Diabetes 54:1684-1691

43. Yokoyama C, Wang X, Briggs MR et al (1993) SREBP-1, a basichelix-loop-helix-leucine zipper protein that controls transcription of the low density lipoprotein receptor gene. Cell 75:187-197

44. Ivan E, Khatri JJ, Johnson C et al (2002) Expansive arterial remodeling is associated with increased neointimal macrophage foam cell content: the murine model of macrophage-rich carotid artery lesions. Circulation 105:2686-2691

45. Watt V, Chamberlain J, Steiner T, Francis S, Crossman D (2011) TRAIL attenuates the development of atherosclerosis in apolipoprotein E deficient mice. Atherosclerosis 215:348-354

46. Kanter JE, Johansson F, LeBoeuf RC, Bornfeldt KE (2007) Do glucose and lipids exert independent effects on atherosclerotic lesion initiation or progression to advanced plaques? Circ Res 100:769-781

47. Johansson F, Kramer F, Barnhart S et al (2008) Type 1 diabetes promotes disruption of advanced atherosclerotic lesions in LDL receptor-deficient mice. Proc Natl Acad Sci U S A 105:2082-2087 